\title{
Utility of Borrelia burgdorferi sensu stricto C6 Peptide for Serologic Confirmation of Erythema-Free Ixodid Tick-Borne Borrelioses in Russia
}

\author{
Vera Pomelova $\mathbb{D}^{1},{ }^{1}$ Eduard Korenberg, ${ }^{2}$ Tatiana Kuznetsova, ${ }^{3}$ and Nikolay Osin ${ }^{4}$ \\ ${ }^{1}$ Laboratory of Molecular Diagnostics, Department of Biological Microassay, State Research Institute of Biological Engineering, \\ 75/1 Volokolamskoye Highway, Moscow 125424, Russia \\ ${ }^{2}$ Department of Infections with Natural Focality, N.F. Gamaleya Research Institute of Epidemiology and Microbiology, \\ 18 Gamaleya Street, Moscow 123098, Russia \\ ${ }^{3}$ Laboratory of Clinical Immunology, Perm Krai Clinical Infectious Diseases Hospital, 96 Pushkin Street, Perm 614000, Russia \\ ${ }^{4}$ Immunoscreen Closed Joint Stock Company, 75/1 Volokolamskoye Highway, Moscow 125424, Russia
}

Correspondence should be addressed to Vera Pomelova; v.pomelova@immunoscreen.ru

Received 20 September 2017; Revised 27 December 2017; Accepted 11 June 2018; Published 28 June 2018

Academic Editor: Lachhman das Singla

Copyright (C) 2018 Vera Pomelova et al. This is an open access article distributed under the Creative Commons Attribution License, which permits unrestricted use, distribution, and reproduction in any medium, provided the original work is properly cited.

\begin{abstract}
We evaluated the utility of Borrelia burgdorferi sensu stricto $(\mathrm{Bb})$ peptide C6 for serologic confirmation of Ixodid Tick-Borne Borrelioses (ITBB) in Russia. Serum samples $(\mathrm{N}=1089)$ were from erythema migrans $(\mathrm{EM})(\mathrm{N}=327)$ and the EM-free $(\mathrm{EMF})$ patients $(\mathrm{N}=115)$; in some patients, the disease was accompanied by human granulocytic anaplasmosis or tick-borne encephalitis. The sera were investigated by multiplex phosphorescence analysis (PHOSPHAN) for IgM to $B b$ C6, recombinant OspC and VlsE proteins, and IgG to C6 from $B b, B$. garinii $(B g)$, and $B$. afzelii $(B a)$. Detection of $B b$ C6 IgM/IgG provided effective serologic confirmation of ITBB in both EM and EMF patients early after disease onset. In the EM-free patients, however, this test needed to be supplemented with detection of VlsE IgM in convalescent-phase sera due to delay in development of the antibody responses for C6 IgG. In general, positive PHOSPHAN reactions were observed in $81.9 \%$ and $86.7 \%$ of the EM and EMF patients, respectively, as well as in 59 of $65(90.8 \%)$ patients, whose blood contained B. burgdorferi sensu lato DNA. Additional detection of IgG to Bg C6 or $\mathrm{Ba}$ C6 had no significant contribution to serologic diagnosis of ITBB in both patient groups.
\end{abstract}

\section{Introduction}

Ixodid Tick-Borne Borrelioses (ITBB) are etiologically independent infections of the Lyme Borreliosis group in Russia [1]. They are caused by spirochetes of the Borrelia burgdorferi sensu lato group transmitted by ixodid ticks. Russia is the largest global areal of B. burgdorferi sensu lato [2,3], with ITBB cases recorded in 74 out of 85 administrative regions. The number of confirmed ITBB cases in 2016 reached approximately 6100 , which was equivalent to 4.18 cases per 100,000 population [4].

Clinical manifestations of Lyme Borreliosis in the United States are differed significantly from those in Eurasia [3, 57]. This is largely due to genetic heterogeneity of Borrelia that cause the disease [8]. B. burgdorferi sensu stricto (hereinafter referred to as B. burgdorferi or $B b$ ) is the major causative agent of Lyme disease in North America, with only a few cases recently linked to Borrelia mayonii [9]. In Europe, at least 5 species of Borrelia (B. afzelii, B. garinii, B. burgdorferi, $B$. spielmani, and B. bavariensis) can cause the disease. Other species (B. bissetii, B. lucitaniae, and B. valaisiana) are sometimes identified in patients but not considered important pathogens [3]. On the territory of Russia, the circulation and distribution of B. garinii, B. afzelii, B. lucitaniae, B. valaisiana, $B$. burgdorferi, and other species of Borrelia have been established [10]. Data were obtained that characterized the genetic polymorphism of B. garinii and B. afzelii [11, 12], the most widespread agents in Eurasia, which are etiologic agents of almost all disease cases in both Europe and Russia [3, 7]. The probability of human infections with several species 
of Borrelia (borrelial-borrelial infection) is rather high. The agents of other tick-borne diseases (e.g., tick-borne encephalitis virus or the human granulocytic anaplasmosis agent) can be transmitted after a tick bite, causing a variety of mixed infections in humans (for review [7]).

In Russia, the ITBB disease is registered in medical documentation as "ITBB with erythema migrans" (EM) and "ITBB without EM or EM-free ITBB" (EMF) [5]. Typical EM is the only pathognomonic sign of early ITBB in Russia. In EM patients, the ITBB disease affects the skin, connective tissue, and musculoskeletal, nervous, and cardiovascular systems [3, 5, 6, 13]. In the United States and Europe, EM occurs in $70-90 \%$ of patients while the extracutaneous manifestations are much less frequent [13]. In Russia, the EM-free forms of ITBB can be diagnosed in $20-45 \%$ of patients in the acute period of the disease [5, 14]. The disease in EMF patients manifests somewhat later (after tick bite), than in EM patients. The disease begins acutely with increasing temperature and development of a general infection syndrome, which is often followed by development of organ pathology affecting one or another system [5]. Common clinical manifestations of the erythema-free form of ITBB are neurologic (22\%), arthromyalgic (15\%), flu-like (12\%), cardiovascular (8\%), hepatic $(4 \%)$, and regional lymphadenitis (1\%); a mixed variant, characterized by a combination of several signs and symptoms, occurs most frequently (38\%) [14]. Pronounced clinical polymorphism in the acute period of ITBB provides evidence for dissemination of the pathogen and generalization of infection.

Clinical diagnosis of ITBB in EMF patients is based on the history of a tick bite, presence of mentioned above clinical manifestations consistent with borreliosis $[5,14]$, and laboratory evidence of $B$. burgdorferi sensu lato infection. Confirmation of active infection consisted of amplification of $B$. burgdorferi sensu lato DNA/RNA in blood by speciesspecific PCR and detection of anti-borreliae immunoglobulin (Ig) $\mathrm{M}$ and $\mathrm{G}$ in acute- and/or convalescent-phase serum samples [5].

However, as it was recently discovered, the febrile and systemic symptoms in tick-exposed patients may be also caused by Borrelia miyamotoi [15-18], a spirochete classified in the relapsing fever group; the B. miyamotoi disease (BMD) may present with clinical manifestations mimicking those in EMF patients. Importantly, the two-tiered diagnostic protocols recommended for serologic confirmation of $B$. burgdorferi infection [19] were reactive for IgM (but not IgG) antibodies to B. burgdorferi in convalescent sera from PCR confirmed BMD cases [18]. Moreover, the FDA-approved C6 peptide ELISA, recently proposed as a potential alternative to conventional two-tier testing $[20,21]$, also was regularly positive on convalescent-phase serum samples from Northeastern American patients with B. miyamotoi infection [22]. According to Telford et al. [18], an IgM reactive sample by Lyme ELISA, but not confirmed by immunoblot, may represent a response to $B$. miyamoto $i$ instead of $B$. burgdorferi. Importantly, the convalescent sera from BMD patients rarely demonstrate IgG reactivity to B. burgdorferi antigens [18].

With this in mind, we present here results of using a multiplex method of phosphorescence analysis (PHOSPHAN)
(Immunoscreen, Russia) for the detection of $B b \operatorname{IgM}$ and $\operatorname{IgG}$ to C6 peptide and of IgMs to OspC or VlsE proteins of $B$. burgdorferi sensu lato. A high efficiency of this method for serologic confirmation of ITBB in EM patients was demonstrated by us earlier [23]. The objective of this research was to compare the utility of PHOSPHAN method for the serologic diagnosis of ITBB in EM patients and the EM-free patients, in which the disease with ITBB occurred as monoinfection or was accompanied by coinfections with human granulocytic anaplasmosis (HGA) or tick-borne encephalitis (TBE). To our knowledge, this is the first work that considers the evolution of $\operatorname{IgM}$ and $\operatorname{IgG}$ antibody responses to the C6 peptide and OspC and VlsE proteins of B. burgdorferi sensu lato in erythema-free ITBB patients as well as in EM and EMF patients coinfected with the agents of HGA or TBE.

\section{Materials and Methods}

2.1. Ethics Statement. The research was performed under the State Research Institute of Biological Engineering, approved protocols numbers 2010/3135/11 and 2017/07/16. Case histories of the patients were deidentified prior to investigation, so the patient informed consent was not required.

2.2. Study Design. Serum samples $(\mathrm{N}=1089)$ were collected from 442 patients with EM and the EM-free (EMF) extracutaneous forms of ITBB during the period of May to September of 2010-2011 in a highly endemic region (Perm Krai) located in the cis-Ural part of Russia. The patients were divided into 6 groups according to physician diagnosed clinical form of ITBB (EM or EMF) and the presence of coinfection with HGA or TBE agents. Serum samples from each group were tested separately for $B b$ C6 IgM and IgG; for OspC IgM and VlsE IgM; for Bg C6 IgG and $\mathrm{Ba}$ C6 IgG using the PHOSPHAN method. IgG antibody responses to recombinant proteins were not included in the analysis since they had no significant contribution to the total C6-IgG responses measured in both EM patients and EMF patients (as was shown in preliminary optimization experiments).

2.3. Case Definitions. The clinical diagnosis of ITBB in EM patients $(\mathrm{N}=327)$ was based on the report of a tick bite and the presence of typical erythema migrans, which was sometimes combined with a general infection syndrome; in 42 out of 142 patients (29.6\%) tested in PCR, the clinical diagnosis was confirmed by PCR detection of B. burgdorferi sensu lato DNA in the blood. ITBB monoinfection was diagnosed in 220 EM patients; in 89 and 18 patients, ITBB was accompanied by HGA (confirmed by PCR detection of Anaplasma phagocytophilum DNA in the blood and/or by recombinant ELISA detection of IgM and IgG to A. phagocytophilum in acute- and/or convalescent-phase serum samples) or TBE (confirmed by ELISA detection of IgM to TBE virus in sera), respectively. Most of the EM patients (93.3\%) had single skin lesions which were located in the site of a tick bite, usually on the trunk and extremities. In 5 patients (2.3\%) with EM monoinfection and 17 EM/HGA patients (19.1\%), multiple EM skin lesions (2 to 39) appeared on days 9-16 after disease onset. 
The clinical diagnosis of ITBB in EM-free patients $(\mathrm{N}$ $=115$ ) was based on the report of a tick bite, presence of characteristic clinical manifestations consistent with borreliosis $[5,14]$, and laboratory evidence of $B$. burgdorferi sensu lato infection. The clinical diagnosis was confirmed by PCR detection of $B$. burgdorferi sensu lato DNA in the blood (in 23 out of 46 patients (50.0\%) tested in PCR) and/or by recombinant ELISA detection of anti-borreliae IgM in acuteand/or convalescent-phase serum samples. ITBB monoinfection was diagnosed in 38 of EMF patients; in 44 and 33 patients, ITBB was accompanied by HGA (confirmed by PCR detection of $A$. phagocytophilum DNA in the blood and/or by recombinant ELISA detection of IgM and IgG to A. phagocytophilum in acute- and/or convalescent-phase serum samples) or TBE (confirmed by ELISA detection of IgM to TBE virus in sera), respectively.

All ITBB patients were treated with antibiotics (mainly doxycycline) at first visit to a clinic [5]. The patients with TBE coinfection were injected with specific antiviral immunoglobulin. Serum samples from majority of patients $(88.5 \%)$ were taken two to five times (prior to treatment and at different times of observation).

2.4. PCR. DNA was extracted from the whole blood dried on filter paper (Whatman 903) by using the Sample-NA kit (DNA Technology, Moscow, Russia) according to manufacturer's instructions. Purified DNA isolates were frozen at $-20^{\circ} \mathrm{C}$. Amplification was performed in a Tercic Thermal Cycler (DNA Technology).

B. burgdorferi sensu lato 5S-23S rRNA intergenic spacer was amplified by using nested PCR with two primer pairs (Bb23SN1-Bb23SC1 and IGSb1-IGSa2) [24]. The PCR inhibition possibility was controlled by 150 -bp long fragments of internal standard [25]. DNA isolated from B. burgdorferi sensu stricto B31 was used as a positive control. All positive amplicons (222-255-bp long) were purified with the Wizard PCR Preps DNA Purification System (Promega, Madison, USA) and treated by TrulI (MseI) restriction enzyme (MBI Fermentas, Vilnius, Lithuania). PCR fragments were visualized under UV irradiation after electrophoresis in agarose gels containing ethidium bromide. The genotyping of Borrelia was performed by analysis of restriction fragment length polymorphism of $\operatorname{rrf}(5 S)-\operatorname{rrl}(23 S)$ intergenic spacer amplicons [24].

A. phagocytophilum DNA was amplified by using nested PCR with two primer pairs (ge3alge10r2 and ge9f3-ge2r) targeted the 16S rRNA gene fragments [26]. DNA isolated from A. phagocytophilum was used as a positive control. Reaction products (546-bp long) were analyzed by agarose gel electrophoresis [27]. Only genetic variant 2 of $A$. phagocytophilum was identified in the study region previously [28].

2.5. ELISA. All serum samples $(\mathrm{N}=1089)$ were tested for IgM and IgG to B. burgdorferi sensu lato, A. phagocytophilum, and TBE virus. Anti-borrelial IgM and IgG were detected by ELISA Omnix KS-001 IgM and KS-002 IgG (Omnix, St. Petersburg). The ELISA consisted of a mixture of recombinant antigens of three Borrelia genospecies ( $B b$ B31, Bg Ip90, and $\mathrm{Ba}$ ACA-1) and thus could detect (but not discriminate) specific antibodies against any of these species. IgM and IgG to A. phagocytophilum were detected by recombinant ELISA Omnix KS-010 IgM and KS-011 IgG. Anti-TBE IgM was detected by the qualitative VectoTBE-IgM ELISA kit (Vector, Novosibirsk, Russia).

\subsection{PHOSPHAN}

2.6.1. Control Sera, Peptide, and Recombinant Antigens. "Positive" and "negative" control serum samples as well as C6 peptides (from $B b$ B31, $B g$ Ip90, and $B a$ ACA-1) and recombinant antigens (OspC, VlsE) used in this study were described in detail in our earlier research [23]. The structure of C6 peptides and the mode of their pretreatment before adsorption in microplates were described elsewhere $[29,30]$.

2.6.2. PHOSPHAN Performance. PHOSPHAN was performed in 96-well microplates as described in detail elsewhere [23]. In brief, an array of nine dots (three per antigen) was printed on the well bottoms. The dots contained combinations of $\mathrm{C} 6 \mathrm{Bb} \mathrm{B} 31$, OspC and VlsE (for IgM detection) or C6 Bb B31, C6 Bg Ip90, and C6 Ba ACA-1 (for IgG detection). The results were expressed as values of the Lyme Index ( $L I)$ and were considered positive (IgM or IgG detected) at $L I \geq 1$.

2.7. Statistics. Differences between proportions were considered significant at 2 -tailed $P \leq 0.05$ (Fisher's exact test).

\section{Results}

3.1. Patients. Table 1 demonstrates the clinical and epidemiological characteristics of ITBB patients $(\mathrm{N}=442)$ enrolled in the research. These patients were divided into 6 groups according to the clinical form of ITBB (erythemic or erythema-free) and the presence of coinfection with HGA or TBE. The patients of these groups did not differ significantly for age, sex (except the EMF/HGA patients, among which the number of men was significantly higher than women), and time of admission after onset of the disease. The majority of patients (96.2\%) mentioned a tick bite preceding the illness. Patients with the erythema-free form of ITBB became sick (after tick bite) significantly later (median: 14; 95\% CI: 12; 16) than EM patients (median: 11; 95\% CI: 9; 11). In the blood of EM/HGA patients and the erythema-free patients, the DNA of B. burgdorferi sensu lato (mainly B. garinii) was detected more frequently than in EM patients (mainly $B$. afzelii) (Table 1). These results are consistent with the data on a longer incubation period $[3,14]$ and more intensive hematogenous dissemination of Borrelia in the erythemafree ITBB patients [5] as well as on clinical manifestations associated with B. garinii or B. afzelii infections [13].

In the majority (more than 70\%) of EM patients, including those with HGA and TBE coinfections, the course of the disease was generally mild (Table 1). Typical EM (usually with a diameter of over $10 \mathrm{~cm}$ ) was accompanied in some cases by symptoms and signs of a general infection syndrome. Most (67 to $87 \%$ ) of the erythema-free patients had a moderate course of the disease (Table 1 ) due to development 


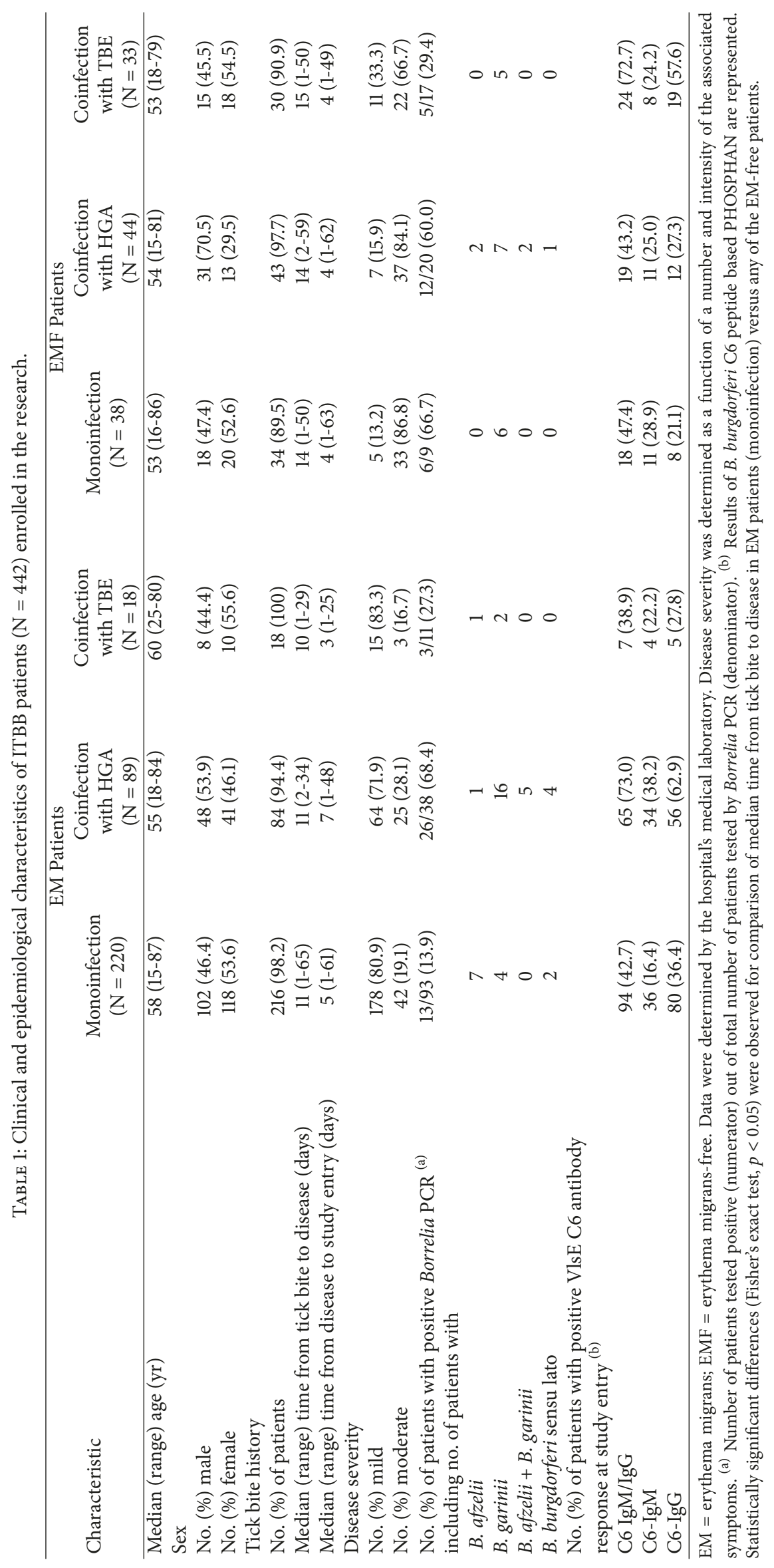


TABLE 2: Sensitivity of B. burgdorferi C6 peptide based PHOSPHAN tests for serum IgM and IgG antibody detection in samples from ITBB patients prior to treatment $(\mathrm{N}=391)$.

\begin{tabular}{|c|c|c|c|c|c|c|c|c|}
\hline \multirow{3}{*}{ Patient Group } & \multicolumn{8}{|c|}{ Number (\%) of positive serum samples at the baseline in ITBB patients as a function of disease duration } \\
\hline & \multicolumn{4}{|c|}{$<7$ days } & \multicolumn{4}{|c|}{$\geq 7$ days } \\
\hline & No. of patients & C6 IgM & C6 IgG & C6 IgM/IgG & No. of patients & C6 IgM & C6 IgG & C6 IgM/IgG \\
\hline EM patients & 133 & $16(12.0)$ & $50(37.6)$ & $56(42.1)$ & 58 & $16(27.6)$ & $30(51.7)$ & $35(60.3)$ \\
\hline EM/HGA patients & 42 & $13(31.0)$ & $21(50.0)$ & $23(54.8)$ & 43 & $19(44.2)$ & $31(72.1)$ & $34(79.1)$ \\
\hline EM/TBE patients & 11 & $2(18.2)$ & $2(18.2)$ & $3(27.3)$ & 6 & $1(16.7)$ & $1(16.7)$ & $2(33.3)$ \\
\hline EMF patients & 24 & $5(20.8)$ & $5(20.8)$ & $8(33.3)$ & 7 & $2(28.6)$ & $2(28.6)$ & $4(57.1)$ \\
\hline EMF/HGA patients & 31 & $3(9.7)$ & $4(12.9)$ & $6(19.4)$ & 9 & $6(66.7)$ & $5(55.6)$ & $7(77.8)$ \\
\hline EMF/TBE patients & 19 & $7(36.8)$ & $8(42.1)$ & $11(57.9)$ & 8 & $1(12.5)$ & $5(62.5)$ & $5(62.5)$ \\
\hline
\end{tabular}

$\mathrm{EM}=$ erythema migrans, EMF = erythema migrans-free, EM/HGA = EM patients coinfected with HGA agent, EM/TBE = EM patients coinfected with TBE virus, $\mathrm{EMF} / \mathrm{HGA}=\mathrm{EMF}$ patients coinfected with HGA agent, and EMF/TBE = EMF patients coinfected with TBE virus. Statistically significant differences (Fisher's exact test, $p<0.05$ ) were observed for comparison of pairs: C6 IgM versus C6 IgG in EM patients at EM duration $<7$ days and $\geq 7$ days; C6 IgM versus C6 IgG in EM/HGA patients at EM duration $\geq 7$ days; C6 IgM at disease duration $<7$ days versus C6 IgM at disease duration $\geq 7$ days in EMF/HGA patients; C6 IgG at disease duration $<7$ days versus C6 IgG at disease duration $\geq 7$ days in EMF/HGA patients. All other comparisons were not statistically significant.

of system and organ pathology [14].At entry to the hospital, positive VlsE C6 antibody responses were detected in 39$73 \%$ of patients in PHOSPHAN tests for total IgM and IgG (C6 IgM/IgG); the lowest index was measured in EM/TBE patients. However, predominant IgG antibody responses to the C6 peptide were not detected in some patients (Table 1), although they were expected [31]. To understand the reasons of this discrepancy and improve the reliability of results, only 391 out of 442 (88.5\%) patients were included in the following research; from each of these individuals two (or more) serum samples were collected (at the entry and at different times of recovery).

3.2. Sensitivity of B. burgdorferi C6 Peptide Based PHOSPHAN. Prior to treatment, the PHOSPHAN positivity for $B b$ C6 IgM and C6 IgG correlated directly with disease duration in all ITBB patients except the EM/TBE and EMF/TBE patients. The number of positive samples was significantly greater in patients with a longer duration of illness ( $\geq 7$ days). Positive PHOSPHAN reaction for C6 IgG was observed more frequently than for C6 IgM only in EM and EM/HGA patients. In all other patients, the number of positive results with $\mathrm{C} 6$ in tests for IgG was about the same as for IgM. The frequency of positive reactions for total $\mathrm{IgM} / \mathrm{IgG}$ exceeded that for C6 IgG alone; however these differences were not statistically significant (Table 2).

Similar patterns of reactivity were measured with C6 peptides from B. garinii and B. afzelii (data not shown). The frequency of positive reactions (for IgG and/or IgM) with these peptides and the results achieved with $B b \mathrm{C} 6$ did not differ significantly (both at the baseline and during the period of observation) in any of the patient groups. Therefore, the results obtained with $\mathrm{Bg} \mathrm{C} 6$ and $\mathrm{Ba} \mathrm{C} 6$ were excluded from further consideration.

3.3. Detection of IgM to OspC and VlsE in PHOSPHAN. Prior to treatment, the PHOSPHAN positivity to OspC and VlsE in tests for IgM correlated directly with disease duration in EM patients as well as in EM/HGA and EMF/HGA patients. In other patients, IgM responses to recombinant proteins were negative (EM/TBE patients) or could be detected irregularly prior to treatment (EMF and EMF/TBE patients) (Table 3).

3.4. The Frequency of IgM and IgG to Borrelia Antigens Detected in Sera from ITBB Patients. Table 4 demonstrates the frequency of anti-Borrelia IgM and IgG antibodies detected in serum samples (prior to treatment and at convalescence) from ITBB patients. Positive PHOSPHAN reactions for C6 IgG were recorded more frequently than for C6 IgM, OspC IgM, or VlsE IgM only in EM and EM/HGA patients. Positive results with any of these antigens were detected significantly more frequently $(p<0.05)$ in serum samples from EM patients with Borrelia-Anaplasma infection than in EM patients with Borrelia monoinfection. In other patients, the number of positive reactions in tests for $\mathrm{C} 6 \mathrm{IgG}$ and $\mathrm{C} 6$ IgM or C6 IgG and VlsE IgM did not differ significantly.

In general, the frequency of positive reactions for C6 IgM/IgG was higher as compared to C6 IgG. However, these differences were statistically significant only in the erythemafree patients. Additional detection of VlsE IgM increased the overall sensitivity of the $\mathrm{C} 6 \mathrm{IgM} / \mathrm{IgG}$ variant; these differences were statistically significant only in EMF/HGA patients.

\subsection{Evolution of Antibody Responses to Borrelia Antigens during the Disease Progression}

3.5.1. EM Patients. In the study of EM patients, the PHOSPHAN results confirmed our earlier observations, obtained on a significantly smaller group of EM patients [23], on the predominant contribution of C6 IgG to the total antibody responses (prior to treatment and at all-time intervals postbaseline) while the contribution of IgMs to $\mathrm{C} 6$, OspC, or VlsE was statistically insignificant $(p>0.05)$. The frequency of detection of IgMs reacting with C6, OspC, or VlsE antigens was low ( $\leq 20 \%)$ at all times of observation (Figure 1(a)).

3.5.2. EM Patients versus EM/HGA Patients. Figure 1(b) shows a similar pattern of antibody responses to Borrelia antigens in EM/HGA patients as compared to EM patients. 


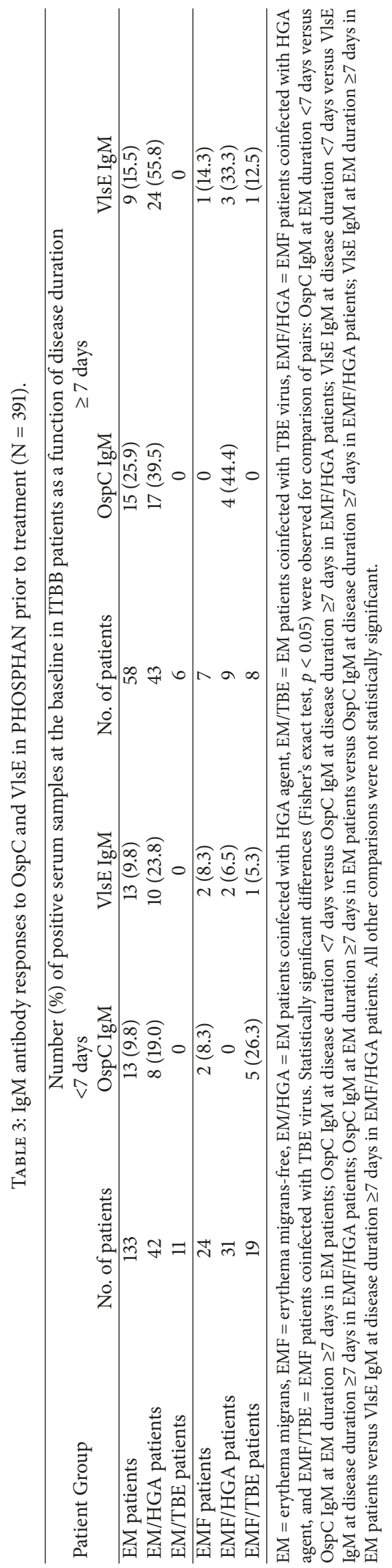


TABLE 4: Frequency of positive antibody responses to Borrelia antigens in serum samples $(\mathrm{N}=1018)$ from ITBB patients.

\begin{tabular}{lccccccc}
\hline \multirow{2}{*}{ Patient Group } & \multirow{2}{*}{ No. of sera } & \multicolumn{5}{c}{ Number (\%) of positive sera (at the baseline and convalescent) to Borrelia antigens } \\
& & OspC IgM & VlsE IgM & C6 IgM & C6 IgG & C6 IgM/IgG & C6 IgM/IgG + VlsE IgM \\
\hline EM patients & 498 & $78(15.7)$ & $65(13.1)$ & $87(17.5)$ & $241(48.4)$ & $266(53.4)$ & $267(53.6)$ \\
EM/HGA patients & 215 & $95(44.2)$ & $117(54.4)$ & $101(47.0)$ & $172(80.0)$ & $177(82.3)$ & $182(84.7)$ \\
EM/TBE patients & 43 & $4(9.3)$ & $6(14.0)$ & $9(20.9)$ & $13(30.2)$ & $17(39.5)$ & $20(46.5)$ \\
\hline EMF patients & 81 & $7(8.6)$ & $26(32.1)$ & $28(34.6)$ & $23(28.4)$ & $45(55.6)$ & $51(63.0)$ \\
EMF/HGA patients & 106 & $24(22.6)$ & $44(41.5)$ & $36(34.0)$ & $30(28.3)$ & $48(45.3)$ & $66(62.3)$ \\
EMF/TBE patients & 75 & $15(20.0)$ & $20(26.7)$ & $27(36.0)$ & $37(49.3)$ & $50(66.7)$ & $59(78.7)$ \\
\hline
\end{tabular}

$\mathrm{EM}=$ erythema migrans, $\mathrm{EMF}=$ erythema migrans-free, $\mathrm{EM} / \mathrm{HGA}=\mathrm{EM}$ patients coinfected with HGA agent, EM/TBE $=\mathrm{EM}$ patients coinfected with TBE virus, $\mathrm{EMF} / \mathrm{HGA}=\mathrm{EMF}$ patients coinfected with $\mathrm{HGA}$ agent, and EMF/TBE = EMF patients coinfected with TBE virus. Statistically significant differences (Fisher's exact test, $\mathrm{p}<0.05$ ) were observed for comparison of pairs: C6 IgG versus OspC IgM, VlsE IgM, and C6 IgM in EM patients; C6 IgG versus OspC IgM, VlsE IgM, and C6 IgM in EM/HGA patients; C6 IgG versus OspC IgM in EMF patients; C6 IgG versus OspC IgM and VlsE IgM in EMF/TBE patients; C6 IgM/IgG versus C6 IgG in EMF/HGA patients; C6 IgM/IgG versus C6 IgG in EMF/TBE patients; C6 IgM/IgG+VlsE IgM versus C6 IgM/IgG in EMF/HGA patients; C6 IgG in EM/HGA patients versus C6 IgG in other groups of patients; C6 IgM in EM/HGA patients versus C6 IgM in EM patients and EM/TBE patients; $\mathrm{C} 6 \mathrm{IgM} / \mathrm{IgG}$ in EM/HGA patients versus $\mathrm{C} 6 \mathrm{IgM} / \operatorname{IgG}$ in other groups of patients. All other comparisons were not statistically significant.

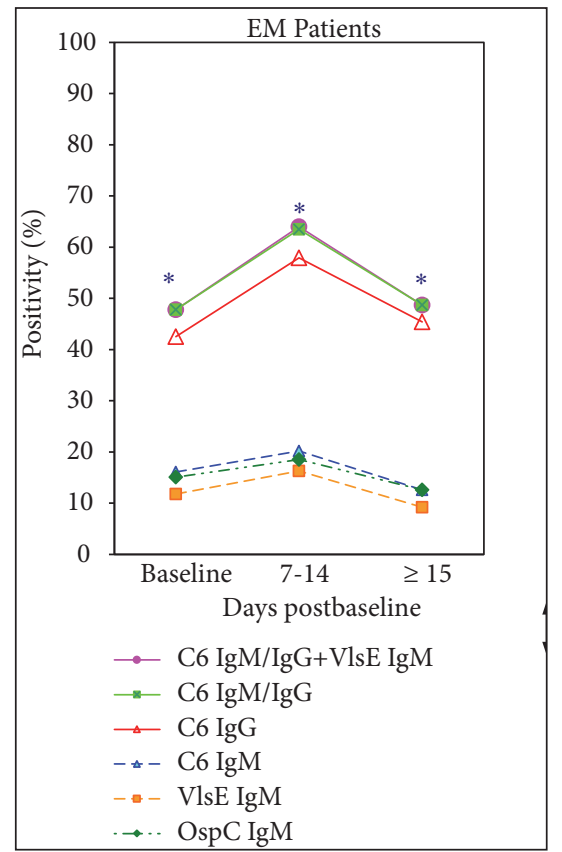

(a) Sensitivity of PHOSPHAN tests detecting IgM and IgG to Borrelia antigens in sera from EM patients (except serum samples from patients with multiple EM skin lesions). The samples were taken prior to treatment $(\mathrm{N}=186)$ and on days $7-14(\mathrm{~N}=178)$ and $\geq 15(\mathrm{~N}=119)$ after disease onset. Statistically significant differences (Fisher's exact test, $\mathrm{p}<0.05)$ were observed for comparison of pairs: C6 IgG versus C6 IgM, OspC IgM, or VlsE IgM prior to treatment and at different times postbaseline. These values are denoted as *

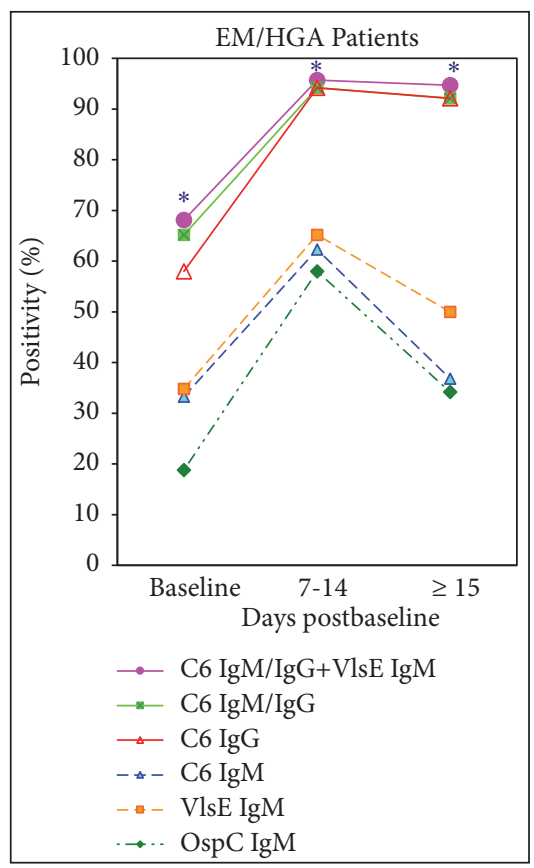

(b) Sensitivity of PHOSPHAN tests detecting IgM and IgG to Borrelia antigens in sera from EM patients coinfected with HGA agent (except serum samples from patients with multiple EM skin lesions). The samples were taken prior to treatment $(\mathrm{N}=69)$ and on days 7-14 $(\mathrm{N}=69)$ and $\geq 15(N=37)$ after disease onset. Statistically significant differences (Fisher's exact test, $\mathrm{p}<$ $0.05)$ were observed for comparison of pairs: C6 IgG versus C6 IgM, OspC IgM, or VlsE IgM prior to treatment and at different times of observation. These values are denoted as $*$

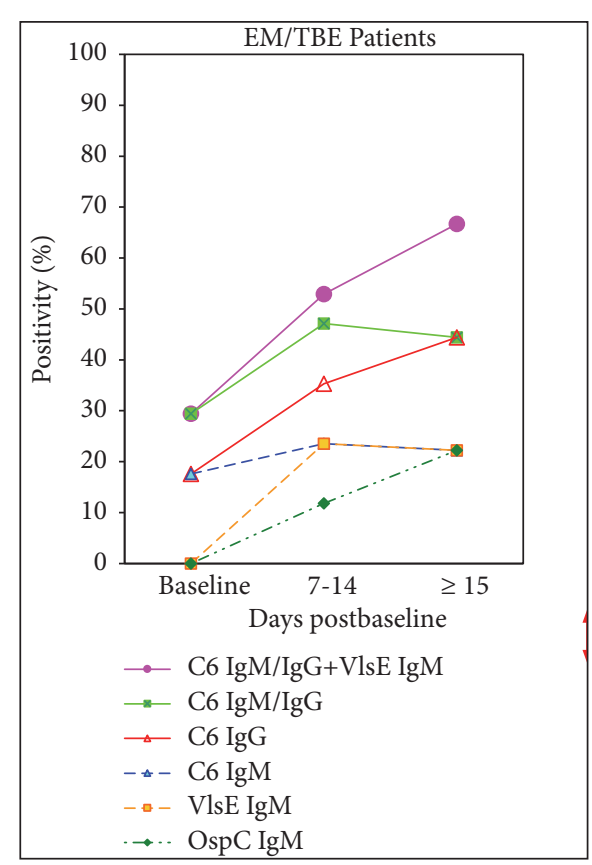

(c) Sensitivity of PHOSPHAN tests detecting IgM and IgG to Borrelia antigens in sera from EM patients coinfected with TBE virus. The samples were taken prior to treatment $(\mathrm{N}=17)$ and on days $7-14(\mathrm{~N}=$ 17) and $\geq 15(\mathrm{~N}=9)$ after disease onset. Statistically significant differences were not observed

Figure 1

At the baseline, the proportion of positive samples for C6 IgM/IgG was slightly higher than for C6-IgG; in later periods of observation, the frequency of these two variants was identical. Maximum frequency of positive responses for C6 $\operatorname{IgG}(94.2 \%)$ and for IgMs (58-65.2\%) was observed on days
7-14 after start of treatment. At later time intervals, the percentage of positive samples with IgMs decreased more rapidly than with C6-IgG (Figure 1(b)).

The frequency of positive reactions for C6 IgM, C6 IgG, and VlsE IgM in the EM/HGA patients was significantly 


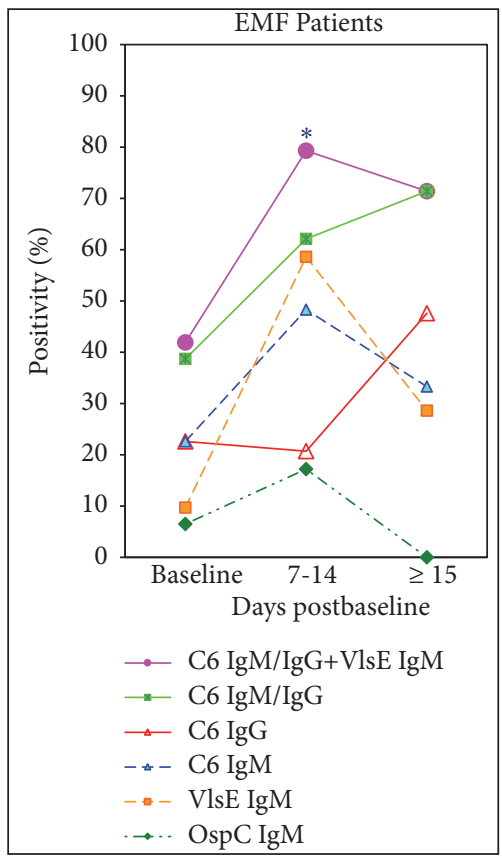

(a) Sensitivity of PHOSPHAN tests detecting IgM and IgG to Borrelia antigens in sera from EM-free patients. The samples were taken prior to treatment $(\mathrm{N}=31)$ and on days $7-14$ $(\mathrm{N}=29)$ and $\geq 15(\mathrm{~N}=21)$ after disease onset. Statistically significant differences (Fisher's exact test, $\mathrm{p}<0.05$ ) were observed for comparison of pairs: C6 IgM/IgG versus C6 IgG and OspC IgM at days 7-14 postbaseline; C6 $\operatorname{IgM} / \mathrm{IgG}+\mathrm{VlsE} \operatorname{IgM}$ versus C6 IgG and OspC IgM at days 7-14 postbaseline. These values are denoted as $*$

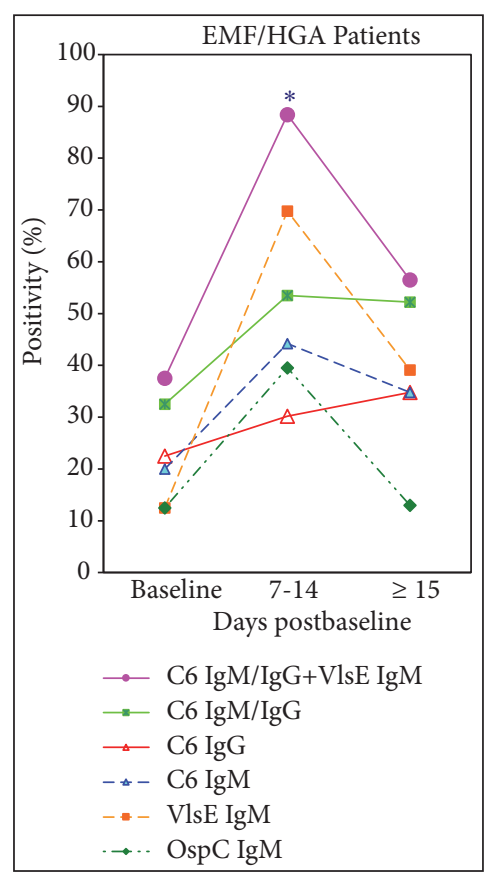

(b) Sensitivity of PHOSPHAN tests detecting $\operatorname{IgM}$ and $\operatorname{IgG}$ to Borrelia antigens in sera from EM-free patients coinfected with HGA agent. The samples were taken prior to treatment $(\mathrm{N}=40)$ and on days 7-14 $(\mathrm{N}$ $=43)$ and $\geq 15(\mathrm{~N}=23)$ after disease onset. Statistically significant differences (Fisher's exact test, $p<0.05$ ) were observed for comparison of pairs: C6 IgM/IgG+VlsE IgM versus C6 IgM/IgG, C6 IgG, C6 IgM, or OspC IgM at days 7-14 postbaseline. These values are denoted as *

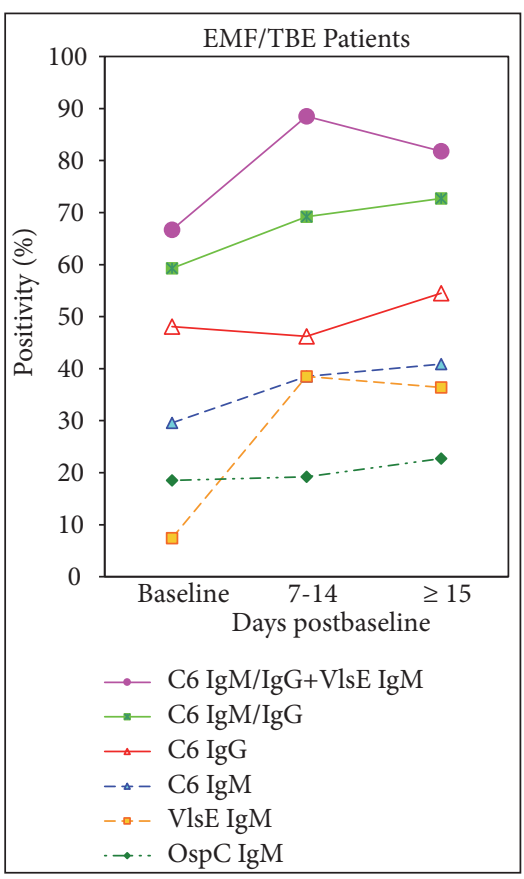

(c) Sensitivity of PHOSPHAN tests detecting IgM and IgG to Borrelia antigens in sera from EM-free patients coinfected with TBE virus. The samples were taken prior to treatment $(\mathrm{N}$ $=27)$ and on days $7-14(\mathrm{~N}=26)$ and $\geq 15(\mathrm{~N}=$ 22) after disease onset. Statistically significant differences were not observed

Figure 2

$(p<0.05)$ higher than in EM patients (both at the baseline and at all times of recovery); differences in the frequency of OspC IgM detection in EM/HGA and EM patients were not statistically significant.

3.5.3. EM Patients versus EM/TBE Patients. Figure 1(c) illustrates a delay in development of the antibody responses in EM/TBE patients. Prior to treatment, the frequency of positive reactions for C6 IgM and C6 IgG was equally low (17.6\%) while the results with OspC and VlsE in tests for IgM were negative. During the disease progression, the frequency of positive results with the C6 peptide in tests for IgG was gradually increased (up to $44 \%$ ). IgMs to OspC and VlsE were detected on days 7-14 and later periods; the frequency of positive reactions to these antigens did not exceed $22 \%$.

In general, the number of positive C6 IgM/IgG responses was insignificantly higher than of C6 IgG alone. Additional detection of VlsE IgM at later time intervals ( $\geq 15$ days) slightly increased the overall sensitivity of the C6 IgM/IgG tests up to $67 \%$ (Figure 1(c)).

Differences in the frequency of positive reactions to any of Borrelia antigens in EM/TBE and EM patients lacked statistical significance at all-time intervals of observation.
3.5.4. EM Patients versus Erythema-Free Patients. Figure 2(a) shows a different pattern of development of the antibody responses in the erythema-free patients as compared to EM patients. At the baseline, the number of positive results with C6 in tests for IgM and IgG was equally low. On days 714 after start of treatment, the number of positive samples with C6 IgG remained the same while the number of positive sera with C6 IgM increased up to $48.3 \%(p>0.05)$; later on ( $\geq 15$ days), the number of positive reactions for C6 IgG increased up to $48 \%$. The proportion of positive C6 IgM/IgG responses was greater than for IgG alone but the difference was statistically significant only in samples collected on days 7-14 after start of treatment. These data provide evidence for delay in development of IgG antibody responses to the peptide C6 in the EMF patients; this delay is accompanied by simultaneous accumulation of positive C6 IgM and VlsE IgM responses on days 7-14 postbaseline.

In general, positive IgM responses were detected in 6.5-23\% samples from the EMF patients prior to treatment. Maximum frequency of IgMs detection (17.2-58.6\%) was observed on days 7-14 after start of treatment; the maximum value of this parameter was measured for VlsE IgM (the difference was statistically significant for comparison of VlsE 
IgM versus $\mathrm{C} 6 \mathrm{IgG})$. The frequency of positive reactions for C6 IgM/IgG (62.1\%) in this time interval was significantly higher than for C6 IgG (20.7\%) due to significant contribution of the C6 IgM. Additional detection of VlsE IgM on days 7-14 after start of treatment increased the overall sensitivity of the C6 IgM/IgG test up to $79.3 \%$ (Figure 1(c)); however, the difference lacked statistical significance.

The frequency of positive reactions to C6 in tests for IgG was significantly $(p<0.05)$ higher (at the baseline and on days 7-14 after treatment) in EM patients as compared to the erythema-free ITBB patients. In contrast, the proportion of positive C6 IgM and VlsE IgM responses was significantly $(p<0.05)$ higher in the erythema-free patients (at all-time intervals after start of treatment) than in EM patients.

3.5.5. EMF Patients versus EMF/HGA Patients. Figure 2(b) shows a similar pattern of development of the antibody responses in EMF/HGA patients as compared to EMF patients. In general, the proportion of positive C6 IgG and C6 IgM responses was comparable both at the baseline (20.0$22.5 \%$ ) and at all-time intervals postbaseline. The number of positive C6 IgM/IgG responses exceeded that for C6 IgG at all times of observation; however, the difference lacked statistical significance.

The maximum number of positive responses for IgMs (39.5-69.8\%) was observed on days 7-14 after start of treatment; the maximum value of this parameter was measured for VlsE IgM (the difference was statistically significant for comparison of VlsE IgM versus C6 IgG). Additional detection of VlsE IgM significantly increased the overall sensitivity of the C6 IgM/IgG test $(p<0.05)$.

3.5.6. EMF Patients versus EMF/TBE Patients. Figure 2(c) shows a similar pattern of development of the antibody responses in EMF/TBE patients as compared to EMF (Figure 2(a)); EMF/HGA (Figure 2(b)) and EM/TBE patients (Figure 1(c)).

The frequency of positive results with the peptide $\mathrm{C} 6$ in tests for IgG was slightly increasing during the disease progression. The proportion of positive C6 IgM/IgG responses exceeded that for C6-IgG at all times of observation; however, the difference lacked statistical significance. Additional detection of VlsE IgM on days 7-14 postbaseline increased the overall sensitivity of the C6 IgM/IgG test up to $89 \%$ ( $p>0.05)$.

Differences in the frequency of positive reactions to any of Borrelia antigens in EMF/TBE and EMF patients lacked statistical significance at all times of observation.

\section{Discussion}

In the present study, we applied a recently developed method of multiplex phosphorescence analysis to detect specific antibody responses to a number of Borrelia antigens. The advantages of PHOSPHAN method as compared to ELISA and immunoblot techniques were described in detail elsewhere [23].

Serum samples for this study were collected in Perm Krai, which is one of the highly endemic regions of Russia.
According to laboratory testing of approximately 500 patients from this area, who became ill during the period of seasonal activity of ticks in 2010, the disease with ITBB was confirmed in $45 \%$ of cases; TBE (10.4\%), HGA (5\%), and human monocytic ehrlichiosis (1.7\%) cases were less frequent; mixed infections were identified in $27 \%$ of patients [32]. These data provide evidence for high pretest probability of ITBB in this region, in combination with other tick-borne infections in particular. In the study region, no research has been performed on the prevalence of B. miyamotoi in tick and human samples, which could confirm a possible role of this spirochete in etiology of ITBB in EMF patients.

One of the most promising approaches to early serologic diagnosis of ITBB caused by B. burgdorferi sensu lato is based on the use of the peptide C6 from VlsE protein of $B$. burgdorferi [31]. The commercial test system (Immunetics C6 ELISA, USA) detects total IgM and IgG antibodies to this peptide, although the main contribution is provided by IgG [31]. We confirmed the effectiveness of this approach in EM patients in Russia [23]. In the present study, we compared the efficiency of the C6 peptide based PHOSPHAN for serologic confirmation of ITBB in Russian patients with EM and the erythema-free manifestations of the disease, which was accompanied in some patients by the ongoing coinfections with HGA or TBE.

Prior to treatment, the frequency of positive results to $B b$ C6 in tests for total IgM/IgG antibodies did not differ significantly in the patient groups. The PHOSPHAN positivity correlated directly with disease duration in all patients except the EM/TBE and EMF/TBE patients (Table 2). Total frequency of positive reactions to the peptide $\mathrm{C} 6$ at different times of recovery did not differ significantly in the groups of patients; however, the values of this parameter were greater in EM/HGA patients, and the lowest values were observed in EM/TBE patients (Table 4, Figures 1 and 2). These results are comparable to the C6 ELISA data [33] and confirm the efficiency of $B b$ C6 IgM/IgG PHOSPHAN assay for serologic confirmation of ITBB in EM and EMF patients early after disease onset.

However, the contribution of IgM and IgG to the total antibody response to $\mathrm{C} 6$ differed significantly in the patient groups.

"Typical" IgM and IgG antibody responses to the peptide C6 [31] were observed only in EM and EM/HGA patients, prior to treatment (Table 2) and at different times of recovery (Table 4, Figures 1(a) and 1(b)). Positive C6 IgG responses were detected significantly more frequently than C6 IgM. Detection of OspC IgM or VlsE IgM did not improve significantly the overall sensitivity of C6 IgM/IgG test, which is consistent with previous data $[23,34]$.

The frequency of positive antibody responses to Borrelia antigens was significantly greater in EM/HGA patients than in EM patients (Table 4, Figures 1(a) and 1(b)). These data, together with positive $B$. burgdorferi sensu lato DNA PCR results in almost 70\% of EM/HGA patients (Table 1), allowed us to suggest that the presence of intracellular infection did not limit the spirochete dissemination.

The intensity of antibody responses to Borrelia antigens was also significantly greater in EM/HGA patients. Median 
$L I$ values for C6 IgG were 32.3 (95\% CI: 12.9; 65.9) versus 7.4 (95\% CI: 4.5; 10.9) in EM/HGA and EM patients with negative B. burgdorferi sensu lato DNA PCR ( $\mathrm{p}<0.05)$, and $66.6(95 \%$ CI: 53 ; 96) versus 8.8 (95\% CI: $3.3 ; 52.5)$ in EM/HGA and EM patients with positive PCR result $(\mathrm{p}<0.05)$. These data provide evidence for a stronger antigenic stimulation of the immunocompetent cells, responsible for production of specific antibodies to Borrelia antigens in EM/HGA patients, and indirectly confirm the spirochete accumulation in the blood of patients with Borrelia-Anaplasma infection, which is consistent with results obtained in the animal models [35-37]. As compared to EM patients with Borrelia monoinfection, clinical manifestations of Borrelia-Anaplasma infection were characterized by a more severe general infection syndrome which was accompanied in some cases by pathology of the internal organs (the liver or less frequently the kidney) [38].

In EM/TBE patients, the proportion of positive C6 IgM and C6 IgG results (prior to treatment) was equally low (Table 2), while the OspC IgM and VlsE IgM antibody responses to OspC and VlsE were negative (Table 3). During disease progression, the frequency of positive reactions to Borrelia antigens gradually increased (Figure 1(c)). Although the number of serum samples at different times of recovery was rather small for making a reliable conclusion, the data obtained confirmed the delay in development of the antibody responses to Borrelia antigens in patients with Borrelia-TBE infection, which also has been noted by other authors; it is believed that the dual infection has no effect on the antibody production against the virus (for review [7]). Active accumulation of IgM to TBE virus in the debut of illness with tick-borne encephalitis [39] as well as the introduction of antiviral immunoglobulin to TBE patients can possibly affect the development of antibody responses to C6 and other Borrelia antigens. Clinical manifestations of Borrelia-TBE virus infections are usually characterized by more acute onset as compared to ITBB patients and more frequent symptoms of general infection syndrome as compared to TBE patients (for review [7]).

In the erythema-free ITBB patients, those with dual Borrelia-Anaplasma and Borrelia-TBE virus infections included, we observed atypical antibody responses to $B$. burgdorferi sensu lato antigens as compared to EM patients. The delay in development of IgG antibody responses to C6 was followed by active accumulation of IgMs to C6, OspC, and VlsE in convalescent-phase serum samples (Figures 2(a), 2(b), and 2(c)). The frequency of positive C6 IgM and C6 IgG reactions (prior to treatment) was comparable (Table 2); however, on days 7-14 postbaseline, the contribution of C6 IgM to the total C6 IgM/IgG antibody response was greater than of C6 IgG (Figures 2(a), 2(b), and $2(\mathrm{c})$ ). Thus, the sensitivity of C6 IgM/IgG PHOSPHAN assay was significantly higher than of C6 IgG alone (Table 4). The maximum number of positive responses for IgMs, to protein VlsE in particular, was observed on days 7-14 after start of treatment (Figures 2(a), 2(b), and 2(c)); additional detection of VlsE IgM increased the overall sensitivity of the C6 IgM/IgG test.

These results provide evidence for modulation of the antibody responses in the erythema-free patients. A significant increase in the level of immunoglobulin $M$ was observed at no change in concentrations of immunoglobulin $G$ and in the cellular component of the immunity [5]. Significant predominance of the IgM response to $B$. burgdorferi and the failure of B cells to undergo class-switch recombination to an IgG response was demonstrated previously in experimental animal models [40]. Importantly, in EMF patients with PCR confirmed B. burgdorferi sensu lato infection, the frequency of positive antibody responses to Borrelia antigens was comparable with the values shown in Table 4, except the fact of more frequent detection of C6 IgG (50-59\% versus 28.3-49.3\%). Additional detection of VlsE IgM increased the overall sensitivity of the C6 IgM/IgG test up to $76-88 \%$. These data possibly indicate an important role of $\operatorname{IgM}$ antibodies in elimination of the pathogen from the blood stream of EMF patients, which was demonstrated in experimental animal models [40].

We can also speculate that at least a part of EMF patients suffered from the ITBB disease caused by $B$. miyamoto $i$ instead of $B$. burgdorferi sensu lato; in patients with PCR confirmed presence of $B$. burgdorferi sensu lato DNA, mixed infection with both agents could occur. This hypothesis, however, is supported only by atypical antibody responses to $B$. burgdorferi sensu lato antigens, which were previously discovered in patients with $B$. miyamotoi disease $[15,17,18$, 22]. Importantly, the $B$. miyamotoi's status as a pathogen has only recently been established. There is still no an adequate and appropriate immunocompetent animal model to study B. miyamotoi infection and identify characteristic symptoms and pathologies of the BMD. The symptoms of this disease are interpreted and extrapolated from complex human cases where disease pathology can be complicated by underlying or unreported medical conditions or coinfections [41].

We can also hypothesize that the erythema-free forms of borreliosis may be caused by other tick-transmitted species of Borrelia, both new and already described, whose role in the etiology of the disease has not yet been adequately proven. Serology developed for Lyme borreliosis can help to confirm an active infection in such patients which provided the crossreactivity between the detected antibodies and $B$. burgdorferi sensu lato antigens [42] which allows for in-time treatment with antibiotics.

The DNA of $B$. garinii was detected in the blood of ITBB patients (except EM patients with ITBB monoinfection) more frequently than $B$. afzelii (Table 1 ). However, positive antibody responses were recorded more frequently with $B b$ C6 or $B a$ C6 than with $B g$ C6 as was shown previously for EM patients [23]. These data confirm that the IR6 region of $B b$ VlsE is highly conserved among European pathogenic genospecies of B. burgdorferi sensu lato [43].

Data on the timing of production of OspC IgM and VlsE IgM, compared to C6 IgM and C6 IgG, in the EMfree patients, as well as in EM and EMF patients with the ongoing HGA and TBE coinfections, are presented here for the first time. Strong C6 IgG and VlsE IgM antibody responses can be considered as important markers of an active Borrelia infection in EM and the erythema-free ITBB patients, respectively. 
The limitation of our study was the fact that the hypothesis on probable B. miyamotoi disease in EMF patients was based just on antibody profile similarity with previously published data. No serologic or PCR tests were performed to confirm this hypothesis.

\section{Conclusion}

The multiplex PHOSPHAN is a promising method for detecting IgM and IgG antibody responses to a number of Borrelia antigens. The detection of $B b C 6 \mathrm{IgM} / \mathrm{IgG}$ provides effective serologic confirmation of ITBB in both EM patients and the erythema-free patients early after disease onset. As the Borrelia infection progressed in the erythema-free patients, the B. burgdorferi $\mathrm{C} 6 \mathrm{IgM} / \mathrm{IgG}$ test needs to be supplemented with detection of VlsE IgM. In general, the PHOSPHAN positivity was recorded in 240 of 293 (81.9\%) patients with erythema migrans and 85 of 98 (86.7\%) patients without cutaneous manifestations of the disease. PHOSPHAN provided serologic confirmation of the disease in 59 of $65(90.8 \%)$ patients, whose blood contained B. burgdorferi sensu lato DNA; only 6 patients tested positive in PCR (5 with EM and 1 without this skin manifestation) were seronegative. The observations from this study document significant differences in the repertoire and kinetics of antibody responses to Borrelia antigens in ITBB patients depending on clinical manifestations (erythemic or erythema-free) of the disease and the presence of coinfection with HGA or TBE agents. Further work is needed to determine the interplay between the antibody responses to Borrelia antigens, primarily to C6 peptide and the parent VlsE molecule, and the underlying mechanisms of innate and adaptive immune responses in both EM and EM-free patients. We also plan to test a possible role of $B$. miyamotoi in etiology of ITBB disease in EMF patients by using the PHOSPHAN immunoassay supplemented with GlpQ protein specific to this pathogen.

\section{Conflicts of Interest}

The authors declare that they have no conflicts of interest.

\section{Acknowledgments}

The authors are grateful to Dr. Barbara J.B. Johnson for providing C6 peptides from B. burgdorferi and B. garinii that were used in this study. They also thank Vladimir Frizen, Natalya Vorob'eva, and Vladimir Teterin for providing the characterized samples of patient sera.

\section{References}

[1] E. I. Korenberg, "Ixodid Tick-borne Borrelioses (ITBB's) - infections of the Lyme Borreliosis group in Russia: country report," in Report of WHO Workshop on Lyme Borreliosis Diagnosis and Surveillance, Warsaw, pp. 128-136, WHO/CDC/VPH/ 95.141-1, 1995.

[2] E. I. Korenberg, N. B. Gorelova, and Y. V. Kovalevskii, Ecology of Borrelia burgdorferi sensu lato in Russia, J. Gray, O. Kahl,
R. S. Lane, and G. Stanek, Eds., Lyme Borreliosis: Biology, Epidemiology and Control. Oxford: CAB International, 2002.

[3] G. Stanek, G. P. Wormser, J. Gray, and F. Strle, "Lyme borreliosis," The Lancet, vol. 379, no. 9814, pp. 461-473, 2012.

[4] State Report, "On the sanitary and epidemiological wellbeing of the population in the Russian Federation in 2016", The Federal service for supervision of consumer rights protection and human welfare, Moscow, Russia, 2017.

[5] N. N. Vorob'eva, Clinical Manifestations, Treatment, and Prophylactics of Ixodid Tick-borne Borrelioses, Ural Press, Perm, Russia, 1998.

[6] G. Stanek, V. Fingerle, K.-P. Hunfeld et al., "Lyme borreliosis: clinical case definitions for diagnosis and management in Europe," Clinical Microbiology and Infection, vol. 17, no. 1, pp. 69-79, 2011.

[7] E. I. Korenberg, V. G. Pomelova, and N. S. Osin, Infections with Natural Focality Transmitted by Ixodid Ticks, Moscow, 2013.

[8] T. Cerar, F. Strle, D. Stupica et al., "Differences in genotype, clinical features, and inflammatory potential of Borrelia burgdorferi sensu stricto strains from Europe and the United States," Emerging Infectious Diseases, vol. 22, no. 5, pp. 818-827, 2016.

[9] B. S. Pritt, P. S. Mead, D. K. H. Johnson et al., "Identification of a novel pathogenic Borrelia species causing Lyme borreliosis with unusually high spirochaetaemia: A descriptive study," The Lancet Infectious Diseases, vol. 16, no. 5, pp. 556-564, 2016.

[10] L. A. Kurkatova, E. I. Korenberg, and N. B. Gorelova, "Modern condition of the domestic museum of Borrelia," Human Health and Environment, vol. 2017, no. 290, pp. 54-56, 2017.

[11] V. V. Nefedova, E. I. Korenberg, and N. B. Gorelova, "Genetic variants of Borrelia garinii, the wide spread agent of infections of the Ixodid Tick-borne Borrelioses group in Eurasia," Molecular Genetics, Microbiology and Virology, vol. 3, pp. 7-12, 2010.

[12] I. A. Fadeeva, V. V. Nefedova, and E. I. Korenberg, "Genetic variants of Borrelia afzelii, one of the agents of infections with Ixodid Tick-borne Borrelioses," Molecular Genetics, Microbiology and Virology, vol. 3, pp. 18-22, 2005.

[13] F. Strle and G. Stanek, Clinical Manifestation and Diagnosis of Lyme Borreliosis, D. Lipsker and B. Jaulhac, Eds., vol. 37, Lyme Borreliosis. Curr Probl Dermatol. Basel: Karger, 2009.

[14] N. N. Vorob'eva and O. N. Sumlivaya, "Clinical variants of Ixodid Tick-borne Borrelioses in the acute period of the disease," Med Parasitology and Parasitary Diseases, vol. 4, pp. 3-7, 2003.

[15] A. E. Platonov, L. S. Karan, N. M. Kolyasnikova et al., "Humans infected with relapsing fever spirochete Borrelia miyamotoi, Russia," Emerging Infectious Diseases, vol. 17, no. 10, pp. 18161823, 2011.

[16] A. E. Platonov, J. Koetsveld, N. M. Kolyashnikova, D. C. Sarksyan, M. G. Toporkova, and G. A. Shipulin, "Microbiological Evidence of etiology of "Ixodes Tick-Borne Borreliosis without Erythema Migrans" - Infection Caused by Borrelia miyamotoi," Epidemiology \& Vaccinal Prevention, vol. 92, no. 1, pp. 29-35, 2017.

[17] P. J. Krause, S. Narasimhan, G. P. Wormser et al., "Borrelia miyamotoi sensu lato seroreactivity and seroprevalence in the northeastern United States," Emerging Infectious Diseases, vol. 20, no. 7, pp. 1183-1190, 2014.

[18] S. R. Telford, H. K. Goethert, P. J. Molloy et al., "Borrelia miyamotoi Disease: Neither Lyme Disease Nor Relapsing Fever," Clinics in Laboratory Medicine, vol. 35, no. 4, pp. 867882, 2015. 
[19] G. P. Wormser, R. J. Dattwyler, E. D. Shapiro, J. J. Halperin, A. C. Steere, M. S. Klempner et al., "The clinical assessment, treatment, and prevention of Lyme disease, human granulocytic anaplasmosis, and babesiosis: Clinical practice guidelines by the Infectious Diseases Society of America," Clinical Infectious Diseases, vol. 43, no. 9, pp. 1089-1134, 2006.

[20] G. P. Wormser, M. Schriefer, M. E. Aguero-Rosenfeld et al., "Single-tier testing with the C6 peptide ELISA kit compared with two-tier testing for Lyme disease," Diagnostic Microbiology and Infectious Disease, vol. 75, no. 1, pp. 9-15, 2013.

[21] G. P. Wormser, A. T. Tang, N. R. Schimmoeller et al., "Utility of serodiagnostics designed for use in the United States for detection of Lyme borreliosis acquired in Europe and vice versa," Medical Microbiology and Immunology, vol. 203, no. 1, pp. 65-71, 2014.

[22] P. J. Molloy, K. E. Weeks, B. Todd, and G. P. Wormser, "Seroreactivity to the C6 Peptide in Borrelia miyamotoi Infections Occurring in the Northeastern United States," Clinical Infectious Diseases, vol. 66, no. 9, pp. 1407-1410, 2018.

[23] V. G. Pomelova, E. I. Korenberg, T. I. Kuznetsova, T. A. Bychenkova, N. I. Bekman, and N. S. Osin, "C6 peptidebased multiplex phosphorescence analysis (PHOSPHAN) for serologic confirmation of lyme borreliosis," PLoS ONE, vol. 10, no. 7, Article ID e0130048, 2015.

[24] D. Postic, M. V. Assous, P. A. D. Grimont, and G. Baranton, "Diversity of Borrelia burgdorferi sensu lato evidenced by restriction fragment length polymorphism of $\operatorname{rrf}(5 S)$-rrl (23S) intergenic spacer amplicons," International Journal of Systematic Bacteriology, vol. 44, no. 4, pp. 743-752, 1994.

[25] A. B. Beklemishev, A. K. Dobrotvorsky, A. V. Piterina, I. D. Ivanov, N. Y. Nomokonova, and N. N. Livanova, "Detection and typing of Borrelia burgdorferi sensu lato genospecies in Ixodes persulcatus ticks in West Siberia, Russia," FEMS Microbiology Letters, vol. 227, no. 2, pp. 157-161, 2003.

[26] R. F. Massung, K. Slater, J. H. Owens et al., "Nested PCR assay for detection of granulocytic ehrlichiae," Journal of Clinical Microbiology, vol. 36, no. 4, pp. 1090-1095, 1998.

[27] V. Y. Teterin, E. I. Korenberg, V. V. Nefedova, N. N. Vorob’eva, and V. V. Frizen, "Enzyme-linked Immunosorbent Assay and Polymerase Chain Reaction in the Laboratory Diagnosis of Human Granulocytic Anaplasmosis," Zh Infektologii, vol. 4, no. 2, pp. 33-39, 2012.

[28] S. Telford, E. Korenberg, H. Goethert, Y. Kovalevskii, N. Gorelova, and A. Spielman, "Detection of Natural Foci of Babesiosis and Granulocytic Ehrlichioses in Russia," Zh Mikrobiol Epidemiol Immunobiol, vol. 6, pp. 21-25, 2002.

[29] H. Sillanpaa, P. Lahdenne, H. Sarvas et al., "Immune responses to borrelial VlsE IR6 peptide variants," International Journal of Medical Microbiology, vol. 297, no. 1, pp. 45-52, 2007.

[30] C. Bieniarz, M. Husain, G. Barnes, C. A. King, and C. J. Welch, "Extended length heterobifunctional coupling agents for protein conjugations," Bioconjugate Chemistry, vol. 7, no. 1, pp. 88-95, 1996.

[31] F. Ting Liang, A. C. Steere, A. R. Marques, B. J. B. Johnson, J. N. Miller, and M. T. Philipp, "Sensitive and specific serodiagnosis of Lyme disease by enzyme-linked immunosorbent assay with a peptide based on an immunodominant conserved region of Borrelia burgdorferi VlsE," Journal of Clinical Microbiology, vol. 37, no. 12, pp. 3990-3996, 1999.

[32] V. Y. Teterin, E. I. Korenberg, V. V. Nefedova et al., "Clinical and laboratory diagnosis of infections transmitted by Ixodes ticks in the Perm Krai," Epidemiology and infectious diseases, vol. no. 4, p. 11, 2013.

[33] T. I. Kuznetsova, V. G. Pomelova, E. I. Korenberg, and N. S. Osin, "The use of multiplex phosphorescence analysis (PHOSPHAN) and polymerase chain reaction for laboratory diagnosis of ixodid tick-borne borrelioses," Epidemiology \& Vaccinal Prevention, vol. 81, no. 2, pp. 38-44, 2015.

[34] M. E. Embers, M. B. Jacobs, B. J. B. Johnson, and M. T. Philipp, "Dominant epitopes of the C6 diagnostic peptide of Borrelia burgdorferi are largely inaccessible to antibody on the parent VlsE molecule," Clinical and Vaccine Immunology, vol. 14, no. 8, pp. 931-936, 2007.

[35] N. S. Zeidner, M. C. Dolan, R. Massung, J. Piesman, and D. Fish, "Coinfection with Borrelia burgdorferi and the agent of human granulocytic ehrlichiosis suppresses IL- 2 and IFN $\gamma$ production and promotes an IL-4 response in $\mathrm{C} 3 \mathrm{H} / \mathrm{HeJ}$ mice," Parasite Immunology, vol. 22, no. 11, pp. 581-588, 2000.

[36] V. Thomas, J. Anguita, S. W. Barthold, and E. Fikrig, "Coinfection with Borrelia burgdorferi and the agent of human granulocytic ehrlichiosis alters murine immune responses, pathogen burden, and severity of lyme arthritis," Infection and Immunity, vol. 69 , no. 5, pp. 3359-3371, 2001.

[37] K. Holden, E. Hodzic, S. Feng, K. J. Freet, R. B. Lefebvre, and S. W. Barthold, "Coinfection with Anaplasma phagocytophilum alters Borrelia burgdorferi population distribution in $\mathrm{C} 3 \mathrm{H} / \mathrm{HeN}$ mice," Infection and Immunity, vol. 73, no. 6, pp. 3440-3444, 2005.

[38] V. Y. Teterin, E. I. Korenberg, V. V. Nefedova, N. N. Vorob’eva, V. I. Frizen, and M. V. Yakusheva, "Human granulocytic anaplasmosis in the Cisural region: mono- and mixed infections with ixodid tick-borne borreliosis," Infectious Diseases, vol. no. 1, pp. 21-27, 2012.

[39] G. Günther, M. Haglund, L. Lindquist, B. Sköldenberg, and M. Forsgren, "Intrathecal IgM, IgA and IgG antibody response in tick-borne encephalitis. Long-term follow-up related to clinical course and outcome," Clinical and Diagnostic Virology, vol. 8, no. 1, pp. 17-29, 1997.

[40] C. J. Hastey, R. A. Elsner, S. W. Barthold, and N. Baumgarth, "Delays and diversions mark the development of B cell responses to Borrelia burgdorferi infection," The Journal of Immunology, vol. 188, no. 11, pp. 5612-5622, 2012.

[41] B. L. Stone and C. A. Brissette, "Host Immune Evasion by Lyme and Relapsing Fever Borreliae: Findings to Lead Future Studies for Borrelia miyamotoi," Frontiers in Immunology, vol. 8, 2017.

[42] L. A. Magnarelli, J. F. Anderson, and R. C. Johnson, "CrossReactivity in Serological Tests for Lyme Disease and other' Spirochetal Infections," The Journal of Infectious Diseases, vol. 156, no. 1, pp. 183-188, 1987.

[43] F. T. Liang, E. Aberer, M. Cinco et al., "Antigenic conservation of an immunodominant invariable region of the VlsE lipoprotein among European pathogenic genospecies of Borrelia burgdorferi SL," The Journal of Infectious Diseases, vol. 182, no. 5, pp. 1455-1462, 2000. 


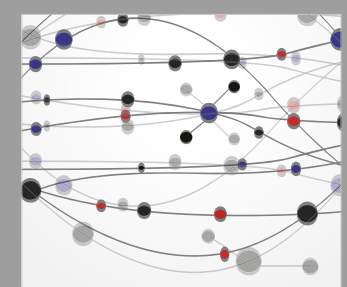

The Scientific World Journal
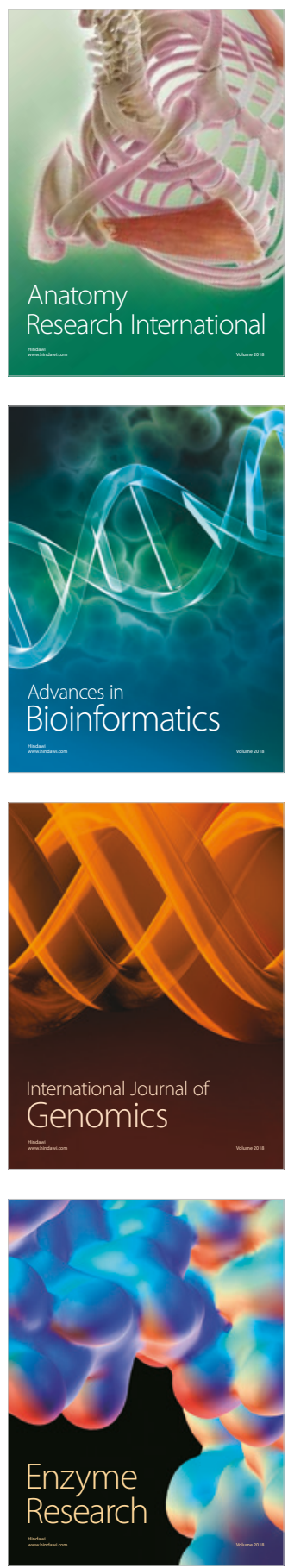
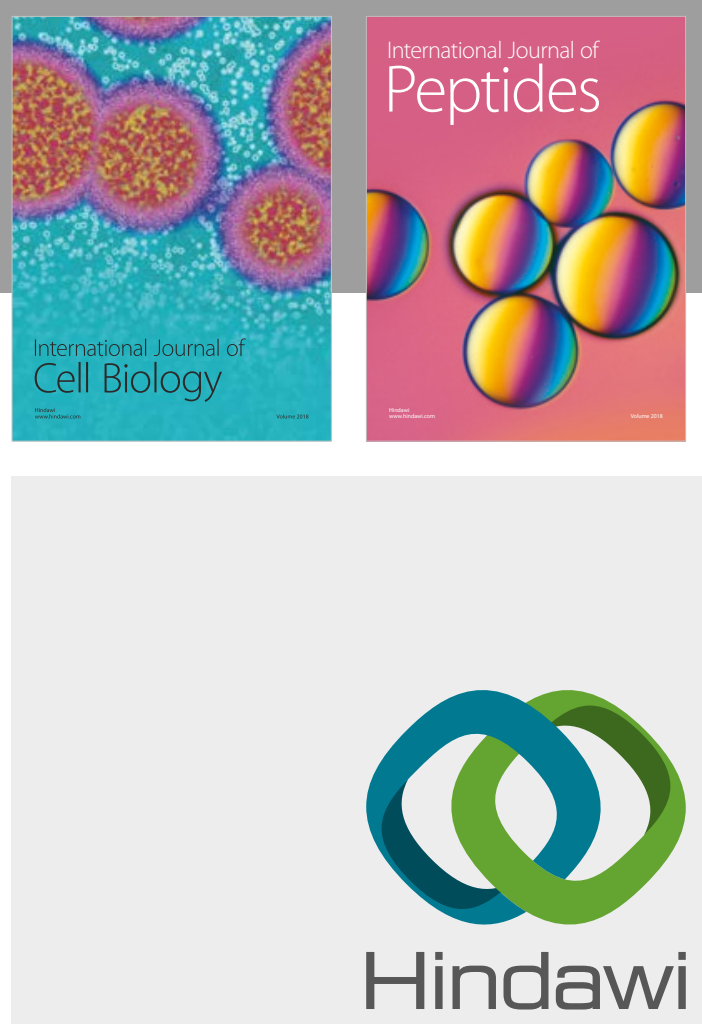

Submit your manuscripts at

www.hindawi.com
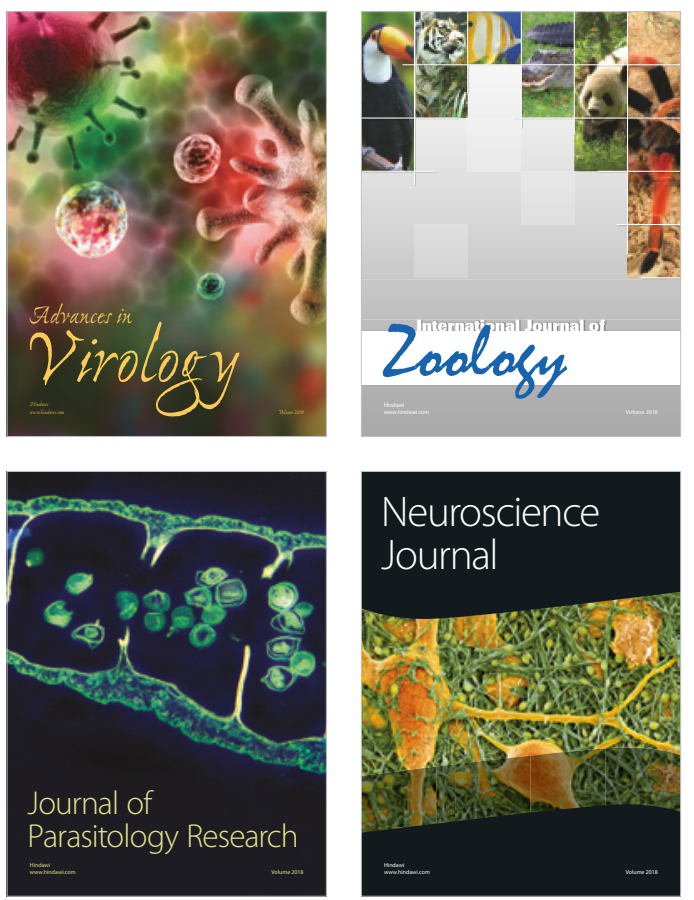
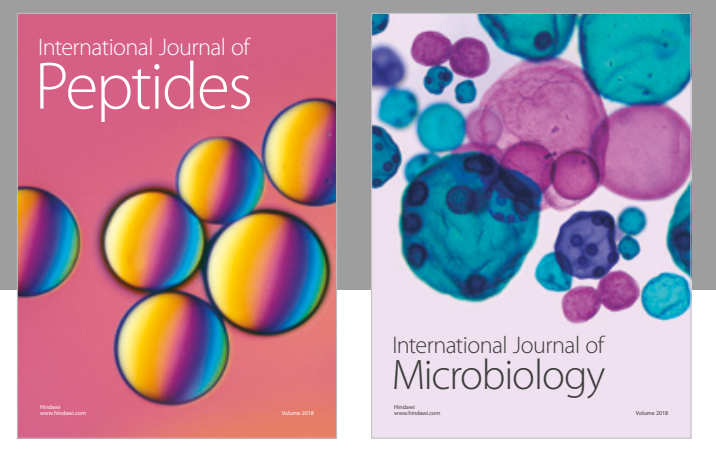

nternational Journal of Microbiology
Journal of
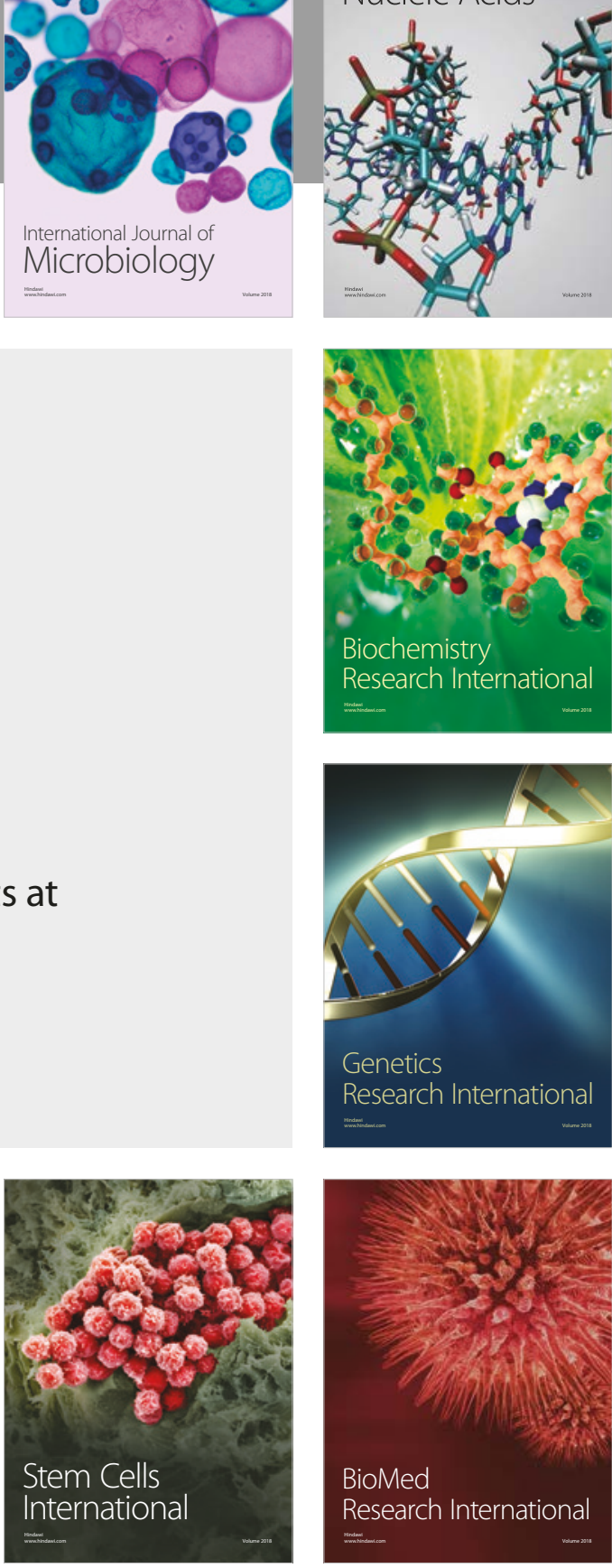
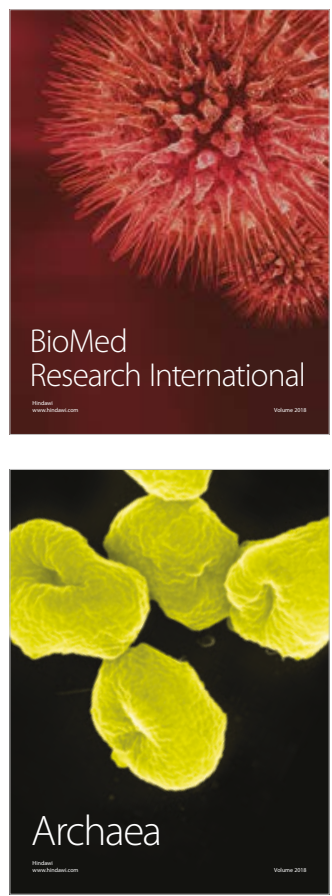